\title{
Effect of orlistat on periostin, adiponectin, inflammatory markers and ultrasound grades of fatty liver in obese NAFLD patients
}

This article was published in the following Dove Press journal:

Therapeutics and Clinical Risk Management

20 February 2017

Number of times this article has been viewed

Rashid Ali Khan'

Prem Kapur ${ }^{2}$

Abhinav Jain ${ }^{3}$

Farrukh Farah ${ }^{4}$

Uma Bhandari'

'Department of Pharmacology, Faculty of Pharmacy, ${ }^{2}$ Department of Medicine, ${ }^{3}$ Department of Radiology,

${ }^{4}$ Department of Paramedical Sciences, Hamdard Institute of Medical Sciences

\& Research, Jamia Hamdard, New

Delhi, India
Correspondence: Uma Bhandari Department of Pharmacology, Faculty of Pharmacy, Jamia Hamdard, Hamdard Nagar, New Delhi I I0062, India Email uma_bora@hotmail.com
Abstract: Orlistat is recommended in the treatment of obesity, which is an independent risk factor for nonalcoholic fatty liver disease (NAFLD). The reported findings of orlistat in NAFLD are divisive. Recently, periostin is identified as an important regulatory molecule in the pathogenesis of obesity-induced fatty liver. Therefore, this study aimed to evaluate the potential effects of orlistat in the treatment of NAFLD. A 16-week prospective observational study was conducted, with obese NAFLD patient $(n=77)$ receiving orlistat (120 mg capsules, three times a day) with hypocaloric diet or hypocaloric diet only. Grades of fatty liver were determined using ultrasound (US) echogenicity of liver; serum levels of periostin, adiponectin, tumor necrosis factor (TNF)- $\alpha$ and interleukin- 6 were determined using ELISA kits at 0 and 16 weeks. Correlations of US grades of fatty liver with these biomarkers were also determined. Orlistat significantly reversed the US grades of fatty liver $(P=0.016)$, decreased serum levels of periostin $(P=0.030)$ and TNF- $\alpha(P=0.040)$, and increased serum adiponectin levels $(P<0.001)$ when compared with hypocaloric diet only. Serum interleukin-6 levels were not found to be significantly different in both groups after the treatment. In the orlistat group, the degree of reduction in grades of fatty liver was found to be positively correlated with the changes in serum levels of periostin $\left(\mathrm{r}_{\mathrm{s}}=0.306, P=0.041\right)$ and adiponectin $\left(\mathrm{r}_{\mathrm{s}}=0.314, P=0.036\right)$, whereas the associations were insignificant with the change in serum levels of TNF- $\alpha\left(r_{s}=0.053, P=0.729\right)$. Mild gastrointestinal side effects $(20 \%)$ were reported in the orlistat group. In conclusion, orlistat is effective in the treatment of NAFLD patients without fibrosis. This study demonstrated a positive association between the reduction of fatty infiltration in the liver and the changes in serum levels of periostin and adiponectin in obese NAFLD patients.

Keywords: NAFLD, orlistat, obesity, periostin, ultrasound grades of fatty liver

\section{Introduction}

Nonalcoholic fatty liver disease (NAFLD) is the most common chronic liver disease encircling a histological scale progressing from simple steatosis to steatohepatitis, and fibrosis. ${ }^{1}$ The global prevalence of NAFLD is $25.24 \%$ with a prevalence in the Indian population ranging from $9 \%$ to $32 \% .^{2,3}$ The precise etiology of NAFLD is unknown; however, there are strong associations with obesity, metabolic/insulin resistance (IR) syndrome and dyslipidemia. ${ }^{4}$ IR is the key factor in the pathogenesis of NAFLD and is deeply entangled with the progression of fatty liver to advanced stages of fibrosis and cirrhosis., ${ }^{5,6}$ A meta-analysis concluded that obesity is a universal, well-reported and independent risk factor for NAFLD. ${ }^{7}$

Current treatment strategies for NAFLD emphasize on the amelioration of risk factors (ie, obesity and IR) with the aim of averting the progression of the disease, or 
regressing established fatty infiltration in the liver. Dietary intervention is an effective weight control strategy for the management of obesity. Very few patients are capable of maintaining reduced body weight and show improvement in fatty liver risk factors through dietary modification alone owing to lack of consistent dietary restrictions. ${ }^{8}$ Till date, there is no approved and established treatment for NAFLD. ${ }^{4,9}$

Orlistat promotes weight loss through reduction of fat absorption from the intestine and has a relatively better side effect profile when compared with other weight loss medications. ${ }^{4,10,11}$ Some evidence show beneficial effects of treatment with this agent in obese patients with NAFLD. ${ }^{12-14}$

Periostin, a matricellular protein, actively contributes to tissue injury, fibrosis, atherosclerosis and inflammatory diseases. Periostin is positively associated with NAFLD, promotes liver steatosis and hypertriglyceridemia, and plays an important role in the progression of steatohepatitis, inflammation and fibrosis. ${ }^{15-17}$ A recent clinical study has suggested that periostin can be used as a potential novel biomarker in the management of NAFLD. ${ }^{18}$

Adiponectin, a protective adipokine, antagonizes hepatic lipid accumulation in addition to insulin-sensitizing, antiinflammatory and antifibrotic effects. Decreased adiponectin levels play a pivotal role in the pathogenesis of NAFLD. ${ }^{19-21}$ Tumor necrosis factor (TNF)- $\alpha$ and interleukin-6 (IL-6), proinflammatory cytokines secreted from various cells/tissues including adipose tissue, also play a vital role in the pathogenesis of NAFLD. ${ }^{22-24}$ Recent clinical studies have suggested that drugs that reduce the levels of serum periostin and inflammatory markers, and elevate serum adiponectin level may provide a better treatment strategy for NAFLD. ${ }^{20,25}$

Thus far, no therapeutic strategies have focused on the downregulation of periostin to improve steatosis, inflammation and fibrosis. This study was aimed to evaluate the effect of orlistat on ultrasound (US) grades of fatty liver, and on serum levels of periostin, adiponectin, TNF- $\alpha$ and IL-6. In addition, study group also attempted to explore the potential correlation between US grades of fatty liver and these biomarkers in obese NAFLD patients.

\section{Materials and methods Study design}

This prospective observational study was conducted over a 16-week period in a clinical setting at the Hamdard Institute of Medical Sciences \& Research (HIMSR), Jamia Hamdard, New Delhi, India, from April 2015 to September 2016. After obtaining approval from the Jamia Hamdard Institutional
Ethics Committee (JHIEC), India, the study was conducted in agreement with the principles enumerated in the Declaration of Helsinki (Brazil, October 2013), ${ }^{26}$ and adhered to the WHO standards for observational studies. The statement for Strengthening the Reporting of Observational Studies in Epidemiology (STROBE) was strictly followed in reporting observations for this study (STROBE checklist, Table S1). ${ }^{27}$ Patients were enrolled in this study only after obtaining written consent through an informed consent form (Figure 1).

\section{Patient recruitment}

Obese NAFLD patients were identified through laboratory investigations, clinical findings and US imaging. Treatments (orlistat, $120 \mathrm{mg}$, three times a day with hypocaloric diet or hypocaloric diet only) were given to obese, adult NAFLD patients of both genders as decided by the treating clinician along with the guidance of a dietician. Based on the intervention, the patients were enrolled in the study, either into orlistat treatment group (orlistat with hypocaloric diet) or diet control group (hypocaloric diet only). Patients were excluded from the study for any of the following reasons: pregnant or nursing women; a history of drug abuse; medical history of diabetes, asthma, serious gastrointestinal, heart, kidney, mental and bone diseases; $>4 \mathrm{~kg}$ weight loss within previous 3 months; use of other weight loss treatments, or consumption of other steatosis-causing drugs such as calcium channel blockers, tamoxifen, amiodarone, prednisolone, corticosterone and methotrexate; a known cause for their increased liver enzyme levels such as viral hepatitis (B or C); any history of surgery within the previous 6 months; history of alcohol consumption of $>20 \mathrm{~g}$ /day over the past 2 years, and recent changes in smoking habits (Figure 1). Orlistat intake was confirmed by prescription and through patient interviews during clinic visits.

\section{Sample size calculation}

No previous study have evaluated the effect of orlistat on serum periostin levels, so required sample size was calculated based on the results of a pilot study, in pre- and post-design manner, involving 10 patients at $\alpha=5 \%$. It was determined that 40 patients were required to provide $80 \%$ statistical power and 55 patients were required for $90 \%$ statistical power of the study.

\section{Clinical and biochemical assessment and follow-up}

Basic information such as age, sex, smoking habits and family history of obesity was recorded during the initial 


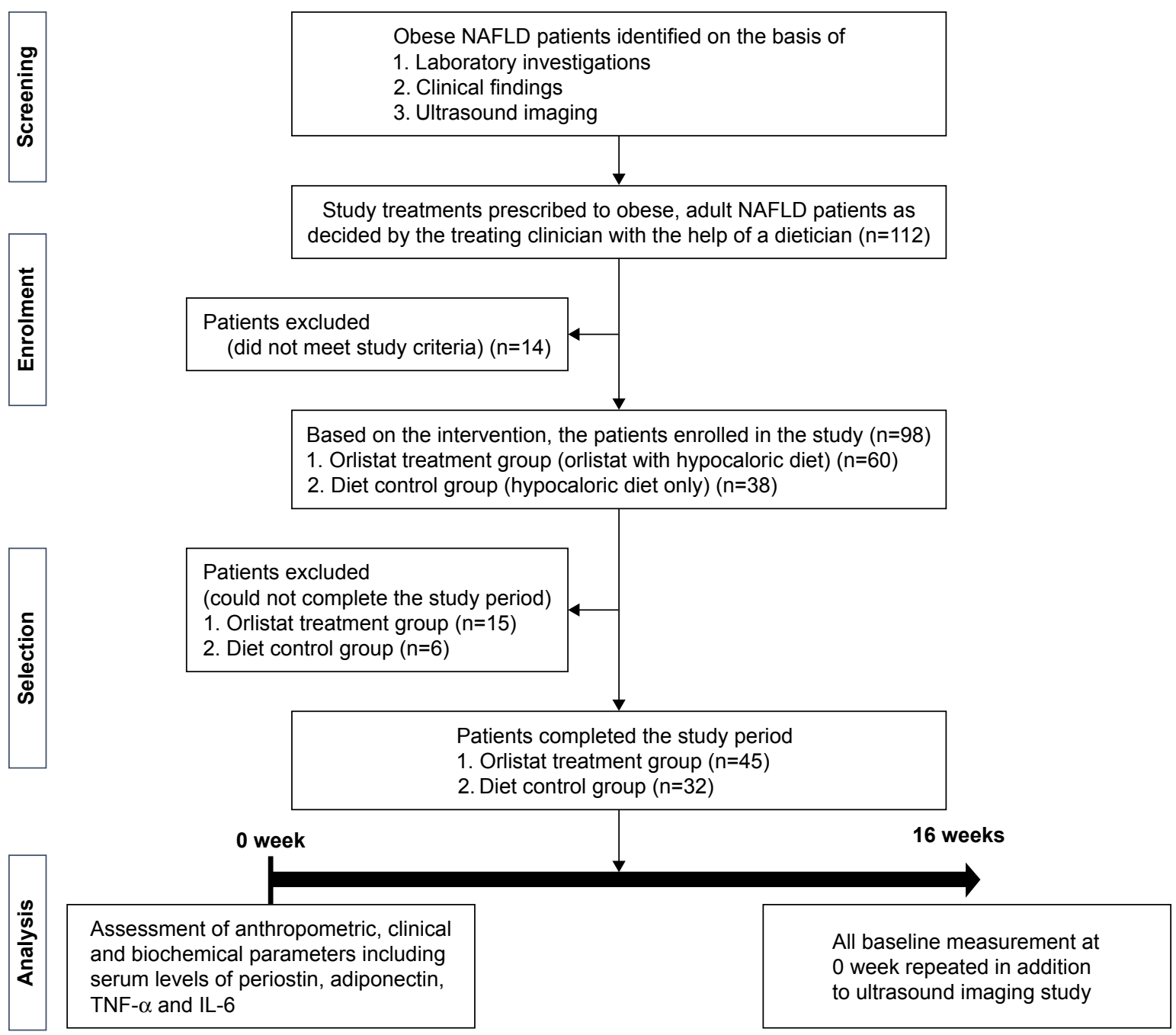

Figure I Study design.

Abbreviations: IL-6, interleukin-6; NAFLD, nonalcoholic fatty liver disease; TNF- $\alpha$, tumor necrosis factor alpha.

visit of the patients. Anthropometric measurements and biochemical parameters, including total cholesterol (TC), triglycerides (TG), low-density lipoprotein (LDL) cholesterol, high-density lipoprotein (HDL) cholesterol, alanine aminotransferase (ALT), aspartate aminotransferase (AST), fasting and postprandial blood glucose levels, glycated hemoglobin $(\mathrm{HbAlc})$, fasting plasma insulin levels, complete blood count including hemoglobin, total leukocyte count, differential leukocyte count, neutrophils, monocytes, lymphocytes, eosinophils, red blood cell count, PCV/hematocrit, mean corpuscular volume, mean corpuscular hemoglobin, mean corpuscular hemoglobin concentration, platelet count and bilirubin, were determined using ELISA kits at the pathology center of HIMSR, New Delhi, at 0 and 16 weeks of orlistat treatment.

A homeostasis model assessment (HOMA) index was used to estimate IR using the following equation: HOMA index $=($ fasting plasma glucose level $[\mathrm{mmol} / \mathrm{L}] \times$ fasting plasma insulin level $[\mu \mathrm{U} / \mathrm{mL}]) / 22.5 .^{28}$ At 0 and 16 weeks of treatment, $5 \mathrm{~mL}$ of blood serum from each enrolled patients was frozen at $-70^{\circ} \mathrm{C}$, and each serum sample was subsequently assessed for periostin, adiponectin, TNF- $\alpha$ and IL-6 levels, using ELISA kits. All estimations were performed in triplicate and were recorded as means \pm standard deviations.

\section{Diet and behavior modifications}

Dietary guidance was given by both dietician and physician. The dietician educated patients about eating behavior including binge eating, control of portion sizes and other adverse habits. The dietician calculated patients' daily calorie requirement using the revised Harris-Benedict equation. ${ }^{29}$ Patients were counseled to follow an individualized diet plan structured as per their hypocaloric diet requirement. Each diet 
chart monitored nutrients, making sure total energy was made up of $55 \%$ carbohydrate, $15 \%$ protein and $30 \%$ fat. Patients were asked to record a 7-day dietary intake and 24-h dietary recall, which were monitored twice in a month either during their clinic visits or through telephone enquiry.

\section{Chemicals}

Laboratory routine test kits were purchased from Roche Diagnostics (Indianapolis, IN, USA, and Mannheim, Germany) and Siemens Healthcare (Frimley, Camberley, UK). Periostin, TNF- $\alpha$ and IL- 6 were measured using ELISA kits from Sincere Biotech (Beijing, People's Republic of China). Adiponectin was measured using ELISA kit from Assaypro (Charles, USA).

\section{Imaging study}

Fatty infiltration in NAFLD was assessed using US echogenicity of liver, by a radiologist, following no $<8 \mathrm{~h}$ of fasting, using a single system GE Voluson S6 US machine with $4 \mathrm{C}$ wide frequency convex transducer at 0 and 16 weeks of study treatments. Grading of fatty infiltrations of the liver was recorded according to standard structure documented in Goldberg textbook. ${ }^{30}$

- Grade 0 (no fatty infiltration) - normal liver echogenicity

- Grade 1 (mild fatty infiltration) - slightly increased echogenicity with standard image of the diaphragm and the intrahepatic vessel borders

- Grade 2 (moderate fatty infiltration) - moderately increased echogenicity with slightly impaired image of the diaphragm or intrahepatic vessels

- Grade 3 (severe fatty infiltration) - markedly increased echogenicity with poor visualization of the diaphragm, the intrahepatic vessels and the posterior portion of the right lobe.

\section{NAFLD fibrosis score and BARD score}

NAFLD fibrosis scores (NFSs) and BARD scores were calculated to identify any advanced fibrosis at 0 and 16 weeks in both groups using the following equations: $\mathrm{NFS}=-1.675+0.037 \times$ age (years) $+0.094 \times$ BMI $\left(\mathrm{kg} / \mathrm{m}^{2}\right)+1.13 \times \mathrm{IFG} /$ diabetes $($ yes $=1$, no $=0)+0.99 \times \mathrm{AST} / \mathrm{ALT}$ ratio $-0.013 \times$ platelet count $\left(\times 10^{9} / \mathrm{L}\right)-0.66 \times$ albumin $(\mathrm{g} / \mathrm{dL})$. A low cut-off (score $<-1.455$ ) excludes advanced fibrosis, whereas a high cut-off (score $>0.676$ ) indicates the presence of advanced fibrosis, and scores between these values are defined as indeterminate. ${ }^{31}$

BARD score was calculated by assigning 0-2 points to the following three variables: AST/ALT ratio $\geq 0.8-2$ points; a body mass index $(\mathrm{BMI}) \geq 28-1$ point; and the presence of diabetes -1 point. Low BARD scores ( 0 or 1$)$ indicate high $(96 \%)$ negative predictive value for advanced fibrosis. ${ }^{32}$

\section{Statistical analysis}

Descriptive statistics were used to analyze the demographic and clinical characteristics of the patients. Data were expressed as mean (SD) or median value (interquartile range). Kolmogorov-Smirnov and Shapiro-Wilk tests were carried out to identify normally distributed variables. Independent sample $t$-tests were used for between-group comparisons at baseline. Paired $t$-tests were used to evaluate within-treatment changes between baseline and the end of treatment, whereas between-treatment changes at the end of treatment were tested using one-way analysis of covariance with the initial value as a covariate. The Wilcoxon signed-rank test, or the Mann-Whitney $U$ test, was used if nonparametric tests were required. Variables were correlated with Spearman's rho correlation analysis. The significance level was set at $P$-value $<0.05$. Data were analyzed using a statistical software SPSS, version 21.0 (SPSS, Inc., Chicago, IL, USA). The sample size was calculated using a software G*Power version 3.1 (Heinrich-Heine-Universität, Düsseldorf, Germany).

\section{Results}

A total of 112 obese NAFLD patients received orlistat and followed hypocaloric diet, or followed a hypocaloric diet only. Patients were screened in accordance with study criteria. At the end of the study, 77 patients $(n=45$, orlistat group; $n=32$, diet control group) completed the study, as analyzed and displayed in Figure 1. The primary reasons of patients for noncompletion of the study in orlistat group were mild gastrointestinal side effects, noncompliance with dietary restriction, financial difficulties, unwillingness to visit the center and commitment toward daily work; whereas switching to drug treatment and noncompliance with dietary restriction were the major reasons for not completing their study in diet control group.

All NAFLD patients were adults (orlistat group vs diet control group; mean age, $40.9 \pm 10.7$ years vs $42.44 \pm 9.23$ years)

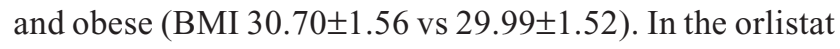
group, 46.66\% were women, compared with $43.75 \%$ women in the control group. There were no significant differences between the groups in demographic, clinical, laboratory, and US imaging characteristics; NAFLD fibrosis scores; and BARD scores at time 0 (Tables 1 and 2). 
Table I Baseline demographics, clinical and laboratory data

\begin{tabular}{|c|c|c|c|}
\hline Variable & Control (32) & Orlistat (45) & $P$-value \\
\hline Age (years) & $42.44 \pm 9.23$ & $40.9 \pm 10.7$ & 0.40 \\
\hline Body weight (kg) & $84.53 \pm 4.75$ & $85.79 \pm 5.46$ & 0.291 \\
\hline BMI $\left(\mathrm{kg} / \mathrm{m}^{2}\right)$ & $29.99 \pm 1.52$ & $30.70 \pm 1.56$ & 0.052 \\
\hline Gender (\%, M/F) & $56.25 / 43.75$ & $53.33 / 46.66$ & 0.803 \\
\hline Smoking (\%) & 12.5 & 13.33 & 0.080 \\
\hline $\begin{array}{l}\text { Family history of } \\
\text { obesity (\%) }\end{array}$ & 15.62 & 17.77 & 0.660 \\
\hline $\mathrm{SBP}(\mathrm{mmHg})$ & $127.0 \pm 13.3$ & $129.0 \pm 9.60$ & 0.257 \\
\hline $\mathrm{DBP}(\mathrm{mmHg})$ & $81.43 \pm 5.63$ & $82.92 \pm 4.66$ & 0.238 \\
\hline Waist (m) & $1.01 \pm 0.04$ & $1.01 \pm 0.05$ & 0.795 \\
\hline Waist/hip ratio & $0.95 \pm 0.03$ & $0.96 \pm 0.021$ & 0.426 \\
\hline FPG (mmol/L) & $4.91 \pm 0.27$ & $4.93 \pm 0.30$ & 0.695 \\
\hline PPG (mmol/L) & $6.69 \pm 0.48$ & $6.47 \pm 0.67$ & 0.125 \\
\hline $\mathrm{HbAlc}(\%)$ & $5.49 \pm 0.25$ & $5.45 \pm 0.24$ & 0.452 \\
\hline Insulin (pmol/L) & $98.66 \pm 22.46$ & $95.97 \pm 24.51$ & 0.625 \\
\hline HOMA-IR & $3.10 \pm 0.75$ & $3.05 \pm 0.86$ & 0.796 \\
\hline $\mathrm{TC}(\mathrm{mmol} / \mathrm{L})$ & $5.56 \pm 0.38$ & $5.30 \pm 0.82$ & 0.099 \\
\hline TG (mmol/L) & $2.14(2.01-2.43)$ & $2.17(1.72-2.21)$ & 0.083 \\
\hline LDL (mmol/L) & $3.61 \pm 0.35$ & $3.42 \pm 0.71$ & 0.163 \\
\hline $\mathrm{HDL}(\mathrm{mmol} / \mathrm{L})$ & $0.91 \pm 0.07$ & $0.90 \pm 0.64$ & 0.632 \\
\hline ALT (ukat/L) & $0.88 \pm 0.21$ & $0.91 \pm 0.21$ & 0.577 \\
\hline AST (ukat/L) & $0.57(0.45-0.61)$ & $0.53(0.47-0.69)$ & 0.872 \\
\hline Bilirubin $(\mu \mathrm{mol} / \mathrm{L})$ & $14.84 \pm 4.56$ & $13.85 \pm 5.66$ & 0.413 \\
\hline Periostin (ng/mL) & $\mathrm{I}, 452.65 \pm 350.83$ & $\mathrm{I}, 479.63 \pm 393.33$ & 0.757 \\
\hline Adiponectin $(\mu g / m L)$ & $5.50 \pm 1.26$ & $5.90 \pm 1.66$ & 0.257 \\
\hline TNF- $\alpha(p g / m L)$ & $6.09 \pm 1.68$ & $6.79 \pm 1.80$ & 0.087 \\
\hline IL-6 (pg/mL) & $4.51 \pm 1.98$ & $3.95 \pm 1.97$ & 0.221 \\
\hline
\end{tabular}

Note: Data are expressed as mean \pm standard deviation, \% or median (interquartile range).

Abbreviations: ALT, alanine aminotransferase; AST, aspartate aminotransferase; BMI, body mass index; DBP, diastolic blood pressure; F, female; FPG, fasting plasma glucose; HbAlc, glycated hemoglobin; HDL, high-density lipoprotein; ukat, unit for catalytic activity; HOMA-IR, homeostasis model assessment-estimated insulin resistance; IL-6, interleukin-6; LDL, low-density lipoprotein; M, male; PPG, postprandial glucose; SBP, systolic blood pressure; TC, total cholesterol; TG, triglyceride; TNF- $\alpha$, tumor necrosis factor alpha.

\section{Treatment outcome Weight loss and waist circumference}

The orlistat group experienced significant greater weight reduction $(6.31 \pm 1.47 \mathrm{vs} 4.07 \pm 0.69 \mathrm{~kg})$ with a marked decrease in waist circumference (WC) as compared to diet control group. However, BMI and waist-to-hip ratio (WHR) were not found to be significantly decreased between both groups after 16 weeks (Table 3 ).

\section{Clinical and biochemical characteristics}

In the orlistat group, most of the metabolic variables showed improvement after 16 weeks of treatment. The serum levels of TC, LDL cholesterol, HDL cholesterol, TG, fasting blood glucose, plasma insulin levels and HOMA-IR values were significantly reduced in orlistat group as compared to the diet control group. Moreover, both groups showed
Table 2 Baseline ultrasound grading and fibrosis scores

\begin{tabular}{llll}
\hline Variable & Control (32) & Orlistat (45) & P-value \\
\hline US grades & $1.78 \pm 0.71$ & $2.02 \pm 0.73$ & 0.14 \\
0 & 0 & 0 & \\
$\mathrm{I}$ & $12(37.5)$ & $\mathrm{II}(24.44)$ & \\
2 & $15(46.87)$ & $22(48.89)$ & \\
3 & $6(15.62)$ & $12(26.67)$ & \\
NFS score & $1.25 \pm 0.44$ & $1.22 \pm 0.42$ & 0.78 \\
Low & $24(75)$ & $35(77.78)$ & \\
Intermediate & $8(25)$ & $10(22.22)$ & \\
High & 0 & 0 & \\
BARD score & $0.91 \pm 0.99$ & $1.20 \pm 0.84$ & 0.052 \\
Low & $26(81.25)$ & $38(84.44)$ & \\
High & $6(18.75)$ & $7(22.22)$ & \\
\hline
\end{tabular}

Note: Data are expressed as $n(\%)$.

Abbreviations: BARD, body mass index plus AST/ALT ratio plus presence of diabetes; NAFLD, nonalcoholic fatty liver disease; NFS, NAFLD fibrosis score; US, ultrasound.

insignificant changes in serum ALT, AST and bilirubin levels (Table 3).

\section{Serum periostin, adiponectin, TNF- $\alpha$ and IL-6}

The orlistat group demonstrated significantly greater reductions in serum periostin (Figure 2) and TNF- $\alpha$ levels, and increase in serum adiponectin levels when compared with the diet control group. There were no significant changes in serum IL-6 levels in either treatment group (Table 3).

\section{US imaging studies}

The orlistat group exhibited more effective reversal of fatty liver infiltration by reducing the US grades of fatty liver when compared with the group treated with hypocaloric diet alone. The US grading of fatty liver was improved in 30 patients $(66.67 \%)$ in orlistat group as compared to 14 patients (43.75\%) of diet control group. Furthermore, US grading of fatty liver was not increased in any patient of orlistat group, whereas the progression of fatty liver by one US grade in four patients (12.5\%), and two grades in one patient (3.12\%) were reported in diet control group (Table 4).

\section{NAFLD fibrosis score and BARD score}

Although orlistat group showed a slight improvement in NFS and BARD scores, but the improvements could not achieve statistical significance after 16 weeks of treatment. NFS scores and BARD scores were also not improved in diet control group (Table 4).

\section{Correlation analysis}

The degrees of reduction in US grade were found to be significantly correlated with weight loss, decreases in serum 
Table 3 Comparison of pre- and postclinical and laboratory variables within and between groups

\begin{tabular}{|c|c|c|c|c|c|c|c|}
\hline Variable & $\begin{array}{l}\text { Control } \\
\text { baseline }\end{array}$ & Control post & $P$-value & $\begin{array}{l}\text { Orlistat } \\
\text { baseline }\end{array}$ & Orlistat post & $P$-value & $\begin{array}{l}P \text {-value } \\
\text { between } \\
\text { groups }\end{array}$ \\
\hline Body weight (kg) & $84.53 \pm 4.75$ & $80.45 \pm 4.55$ & $<0.00 I^{* *}$ & $85.79 \pm 5.46$ & $79.49 \pm 5.29$ & $<0.00 I^{* *}$ & $<0.001^{\$}$ \\
\hline $\operatorname{BMI}\left(\mathrm{kg} / \mathrm{m}^{2}\right)$ & $30 \pm 1.52$ & $28.54 \pm 1.50$ & $<0.00 I^{* *}$ & $30.70 \pm 1.56$ & $28.44 \pm 1.47$ & $<0.00 I^{* *}$ & 0.897 \\
\hline Waist (m) & $1.01 \pm 0.04$ & $1.00 \pm 0.04$ & $<0.00 I^{* *}$ & $1.01 \pm 0.05$ & $0.98 \pm 0.04$ & $<0.00 I^{* *}$ & $<0.00 I^{\$}$ \\
\hline Waist/hip ratio & $0.95 \pm 0.03$ & $0.95 \pm 0.02$ & 0.718 & $0.96 \pm 0.021$ & $0.95 \pm 0.024$ & $0.023^{*}$ & 0.122 \\
\hline FPG (mmol/L) & $4.9 I \pm 0.27$ & $4.85 \pm 0.32$ & 0.139 & $4.93 \pm 0.30$ & $4.81 \pm 0.39$ & $0.011 *$ & $0.010^{\#}$ \\
\hline PPG (mmol/L) & $6.69 \pm 0.48$ & $6.67 \pm 0.57$ & 0.762 & $6.47 \pm 0.67$ & $6.3 I \pm 0.7 \mid$ & 0.066 & 0.080 \\
\hline $\mathrm{HbAlc}(\%)$ & $5.49 \pm 0.25$ & $5.47 \pm 0.31$ & 0.292 & $5.45 \pm 0.24$ & $5.40 \pm 0.28$ & 0.122 & $0.010^{\#}$ \\
\hline Insulin (pmol/L) & $98.66 \pm 22.46$ & $97.94 \pm|5.6|$ & 0.853 & $95.97 \pm 24.5$ I & $88.44 \pm 23.79$ & $0.012^{*}$ & $0.042^{\#}$ \\
\hline HOMA-IR & $3.10 \pm 0.75$ & $3.04 \pm 0.56$ & 0.623 & $3.05 \pm 0.86$ & $2.75 \pm 0.84$ & $0.004^{*}$ & $0.048^{\#}$ \\
\hline $\mathrm{TC}(\mathrm{mmol} / \mathrm{L})$ & $5.26 \pm 0.48$ & $5.07 \pm 0.68$ & 0.084 & $5.30 \pm 0.82$ & $5.05 \pm 0.75$ & $0.005^{*}$ & $0.020^{\#}$ \\
\hline TG (mmol/L) & $2.14(2.0 \mathrm{I}-2.43)$ & $2.08(1.8-2.3)$ & 0.022 & $2.17(1.72-2.21)$ & $1.95(1.64-1.95)$ & $<0.001 * *$ & $0.007^{\#}$ \\
\hline LDL (mmol/L) & $3.36 \pm 0.47$ & $3.22 \pm 0.65$ & 0.179 & $3.42 \pm 0.71$ & $126.89 \pm 0.73$ & 0.069 & $<0.001^{\$}$ \\
\hline $\mathrm{HDL}(\mathrm{mmol} / \mathrm{L})$ & $0.91 \pm 0.07$ & $0.90 \pm 0.08$ & 0.515 & $0.90(0.85-0.96)$ & $0.94(0.91-1.01)$ & $<0.00 I^{* *}$ & $0.029^{\#}$ \\
\hline ALT (ukat/L) & $0.88 \pm 0.21$ & $0.83 \pm 0.18$ & 0.103 & $0.91 \pm 0.21$ & $0.87 \pm 0.23$ & 0.207 & 0.540 \\
\hline AST (ukat/L) & $0.57(0.45-0.6 \mathrm{I})$ & $0.54(0.42-0.54)$ & 0.210 & $0.53(0.47-0.69)$ & $0.5 \mathrm{I}(0.42-0.6 \mathrm{I})$ & $0.022^{*}$ & 0.646 \\
\hline Bilirubin $(\mu \mathrm{mol} / \mathrm{L})$ & $14.84 \pm 4.56$ & $|4.9| \pm 5.8 \mid$ & 0.917 & $13.85 \pm 5.66$ & $14.02 \pm 5.69$ & 0.763 & 0.942 \\
\hline Periostin (ng/mL) & $\mathrm{I}, 452.65 \pm 350.83$ & $\mathrm{I}, 446.98 \pm 370.36$ & 0.750 & 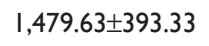 & I,398.97 \pm 379.78 & $0.002 *$ & $0.030^{\#}$ \\
\hline Adiponectin $(\mu \mathrm{g} / \mathrm{mL})$ & $5.50 \pm 1.26$ & $5.7 I \pm 1.33$ & 0.068 & $5.90 \pm 1.66$ & $6.84 \pm 1.80$ & $<0.00 I^{* *}$ & $<0.00 I^{\$}$ \\
\hline TNF- $\alpha(\mathrm{pg} / \mathrm{mL})$ & $6.09 \pm 1.68$ & $6.47 \pm 1.33$ & 0.518 & $6.79 \pm 1.80$ & $6.29 \pm 1.65$ & $0.014^{*}$ & $0.040^{\#}$ \\
\hline IL-6 (pg/mL) & $3.69 \pm 1.29$ & $3.88 \pm 1.32$ & 0.268 & $3.95 \pm 1.97$ & $3.82 \pm 1.95$ & 0.179 & 0.718 \\
\hline
\end{tabular}

Notes: Data are expressed as mean \pm standard deviation, \% or median (interquartile range), ${ }^{* * P} P$-value is $<0.001$ within groups, ${ }^{\$} P$-value is $<0.00 \mathrm{I}$ between groups, $* P$-value is $<0.05$, within groups, " $P$-value is $<0.05$ between groups.

Abbreviations: ALT, alanine aminotransferase; AST, aspartate aminotransferase; BMI, body mass index; DBP, diastolic blood pressure; F, female; FPG, fasting plasma glucose; $\mathrm{HbAlc}$, glycated hemoglobin; HDL, high-density lipoprotein; HOMA-IR, homeostasis model assessment-estimated insulin resistance; IL-6, interleukin-6; LDL, low-density lipoprotein; M, male; PPG, postprandial glucose; SBP, systolic blood pressure; TC, total cholesterol; TG, triglyceride; TNF- $\alpha$, tumor necrosis factor alpha; ukat, unit for catalytic activity.

periostin levels and increases in serum adiponectin levels in the orlistat group. There were no significant associations found between US grade reduction and change in serum TNF- $\alpha$ levels in the orlistat group (Table 5). Moreover,

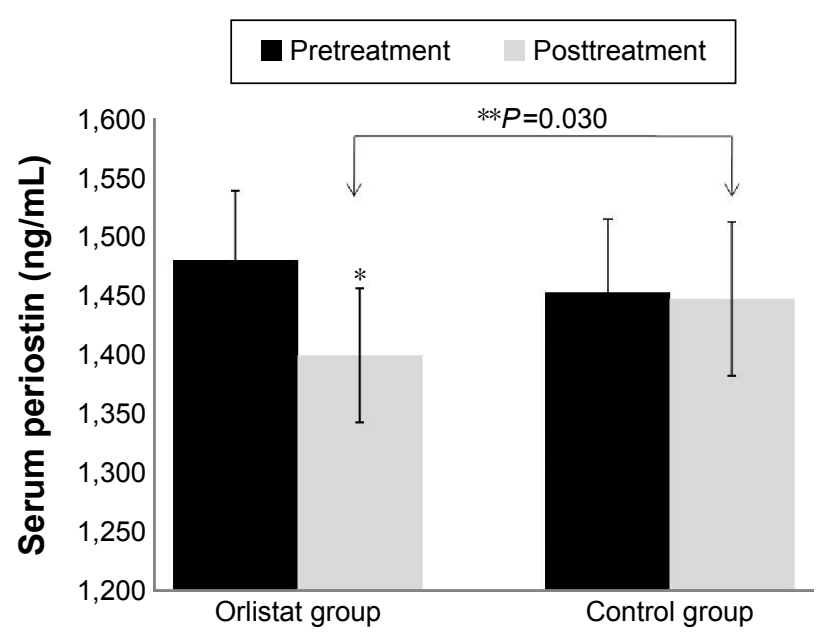

Figure 2 Effect of orlistat on serum periostin level in obese nonalcoholic fatty liver disease patients.

Notes: There was no significant group difference in pretreatment serum periostin levels $(P>0.05)$. $* P<0.05$, value significantly different from baseline value. $* * P<0.05$, value significantly different between groups. orlistat group also showed a positive correlation between decreases in serum levels of periostin and $\mathrm{TG}\left(\mathrm{r}_{\mathrm{s}}=0.319\right.$, $P=0.032$ ), and between weight loss and increases in adiponectin level $\left(\mathrm{r}_{\mathrm{s}}=0.374, P=0.011\right)$.

\section{Discussion}

Orlistat was suggested as a potential drug in the treatment of NAFLD, as it is recommended in the treatment of obesity, which is one of the major independent risk factors of NAFLD. However, the efficacy of orlistat in NAFLD has divisive findings and contrary reports in the previous studies. Though two small uncontrolled clinical studies and one largecontrolled study have shown the effectiveness of orlistat in the reversal of fatty infiltration and improvement in hepatic fibrosis in obese NAFLD patients. ${ }^{12-14}$ However, in another clinical study by Harrison et al, ${ }^{33}$ orlistat was not found to be effective in the treatment of nonalcoholic steatohepatitis (NASH). This study evaluated the role of orlistat in Indian obese NAFLD patients and documented the effect of orlistat on a novel biomarker, periostin, in obese NAFLD patients.

In this study, US of fatty liver after 0 and 16 weeks of orlistat treatment indicated that orlistat was more effective 
Table 4 Comparison of NAFLD US grades, NFS and BARD score within and between groups

\begin{tabular}{|c|c|c|c|c|c|c|c|}
\hline \multirow[t]{2}{*}{ Variable } & \multicolumn{2}{|c|}{ Control (32) } & \multirow[t]{2}{*}{$P$-value } & \multicolumn{2}{|c|}{ Orlistat (45) } & \multirow[t]{2}{*}{$P$-value } & \multirow{2}{*}{$\begin{array}{l}P \text {-value } \\
\text { between } \\
\text { groups }\end{array}$} \\
\hline & 0 week & 16 weeks & & 0 week & 16 weeks & & \\
\hline US grades & I.78 $\pm 0.7 \mid$ & $1.49 \pm 0.80$ & 0.062 & $2.02 \pm 0.73$ & $1.20 \pm 0.94$ & $<0.00 I^{* *}$ & $0.016^{\$}$ \\
\hline 0 & 0 & $2(6.25)$ & & 0 & II (24.44) & & \\
\hline I & $12(37.5)$ & I7 (53.I2) & & II (24.44) & $19(42.22)$ & & \\
\hline 2 & $15(46.87)$ & $9(28.12)$ & & $22(48.89)$ & $10(22.22)$ & & \\
\hline 3 & $6(15.62)$ & $4(12.5)$ & & $12(26.67)$ & $5(11.11)$ & & \\
\hline NFS score & $1.25 \pm 0.44$ & $1.28 \pm 0.46$ & 0.705 & $1.22 \pm 0.42$ & $1.20 \pm 0.40$ & 0.655 & 0.426 \\
\hline Low & $24(75)$ & $23(71.875)$ & & 35 (77.78) & $36(80)$ & & \\
\hline Intermediate & $8(25)$ & $9(28.125)$ & & $10(22.22)$ & $9(20)$ & & \\
\hline High & 0 & 0 & & 0 & 0 & & \\
\hline BARD score & $0.91 \pm 0.99$ & $1.03 \pm 1.06$ & 0.157 & $1.20 \pm 0.84$ & $1.09 \pm 0.87$ & 0.248 & 0.137 \\
\hline Low & $26(81.25)$ & $25(78.12)$ & & 38 (84.44) & 39 (86.67) & & \\
\hline High & $6(18.75)$ & $7(21.875)$ & & $7(22.22)$ & $8(17.78)$ & & \\
\hline
\end{tabular}

Notes: Data are expressed as $\mathrm{n}(\%)$. ${ }^{* *} P$-value is $<0.001$ within groups, ${ }^{\$} P$-value is $<0.05$ between groups.

Abbreviations: ALT, alanine aminotransferase; AST, aspartate aminotransferase; BARD, body mass index plus AST/ALT ratio plus presence of diabetes; NAFLD, nonalcoholic fatty liver disease; NFS, NAFLD fibrosis score; US, ultrasound.

than hypocaloric diet in the reduction of US grades of fatty liver. This is due to the resulting reversal of fatty infiltration in liver. Orlistat also significantly improved metabolic variables including blood glucose level, insulin sensitivity, lipid profiles and liver enzymes. These findings are consistent to those found in previous clinical studies ${ }^{12-14}$ and contrary to those reported by Harrison et al..$^{33}$ This difference could be attributed to the types of subjects that participated in the study by Harrison et al, ${ }^{33}$ who were NASH patients with mean BMI 34, compared with this study wherein NAFLD patients (low NFS and BARD score) with mean BMI 30.70, were enrolled. The patients treated with orlistat showed a significant reversal in steatosis, as evidenced by US grades, but failed to improve fibrosis as insignificant decreases in NFS and BARD scores were observed. This was in agreement with the findings of Harrison et al..$^{33}$ It is well known that development of obesity as well as anti-obesity effect under orlistat treatment depends on dietary nature of patients. Most studies assessing the effects of orlistat on NAFLD have been

Table 5 Correlation of the degree of reduction in ultrasound score with percentage weight loss

\begin{tabular}{llllll}
\hline Factor (\%) & \multicolumn{2}{l}{ Control (32) } & & \multicolumn{2}{c}{ Orlistat (45) } \\
\cline { 2 - 3 } \cline { 6 - 7 } & $\mathbf{r}_{\mathrm{s}}$ & $\boldsymbol{P}$-value & & $\mathbf{r}_{\mathrm{s}}$ & $\boldsymbol{P}$-value \\
\hline Weight loss & 0.377 & $0.034^{*}$ & & 0.331 & $0.026^{*}$ \\
Periostin decrease & 0.275 & 0.178 & & 0.306 & $0.04 I^{*}$ \\
Adiponectin increase & 0.335 & 0.061 & & 0.314 & $0.036^{*}$ \\
TNF- $\alpha$ decrease & 0.019 & 0.919 & & 0.053 & 0.729 \\
IL-6 decrease & -0.017 & 0.926 & & 0.021 & 0.892 \\
\hline
\end{tabular}

Notes: Percentage change in periostin, adiponectin, TNF- $\alpha$ and IL-6 after 16-week treatment of orlistat. $* P$-value is $<0.05$.

Abbreviations: IL-6, interleukin-6; $r_{s}$, Spearman's correlation; TNF- $\alpha$, tumor necrosis factor alpha. performed on European populations, and their diets are different from those of the Indian population. ${ }^{12-14,34}$ Additionally, NAFLD can also be influenced by racial/ethnic background and genetic variation in specific genes. ${ }^{35}$ In this study, we confirmed the beneficial effects of orlistat in Indian obese NAFLD patients without fibrosis.

Periostin plays a pivotal role in the development of obesity-induced hepatosteatosis in obese animal models. ${ }^{16}$ Furthermore, Huang et $\mathrm{al}^{16}$ also demonstrated a positive association between liver fibrosis with the upregulated expression of periostin in an animal model. Zhu et al ${ }^{18}$ confirmed the role of periostin in the pathogenesis of NAFLD in a clinical study, possibly in the regulation of inflammation and insulin sensitivity. In line with the previous studies, ${ }^{15-18}$ this study explored the effect of orlistat on serum periostin levels in NAFLD patients and documented a greater reduction in serum periostin levels in comparison to hypocaloric diet.

Since orlistat is not considered to have direct systemic effect, reduction in serum periostin levels is explained indirectly by its effect on obesity and impaired glucose metabolism as documented by Luo et al, ${ }^{36}$ who reported that periostin levels are positively and cumulatively regulated by obesity and impaired glucose metabolism. Their findings established a positive association between body fat parameters namely BMI, WC and WHR with periostin levels. They further reported strong associations between periostin and TG metabolism, chronic inflammation and IR, and demonstrated that TG, TNF- $\alpha$ and HOMA-IR were independent factors that influenced the periostin levels in Chinese obese patients. ${ }^{36} \mathrm{~A}$ similar association was also reported by Amara et $\mathrm{al}^{17}$ in an animal model. The findings of these studies were 
consistent with those of the current study in which orlistat produced a marked reduction in obesity and its related parameters, and showed notable improvement in impaired glucose parameters. Furthermore, orlistat also improved the levels of TG, TNF- $\alpha$ and HOMA-IR, and showed a positive correlation between levels of periostin and TG levels, which might be the major responsible factors in the downregulation of periostin levels.

Another potential mechanism to explain the effect of orlistat on periostin levels could be the propensity for orlistat to lower circulating lipopolysaccharide (LPS) levels by acting on intestinal microbiota. ${ }^{37}$ This is an important effect considering that LPS induced the periostin level in the animal model of cardiac fibrosis. ${ }^{38}$ The effect of LPS on periostin levels has not yet been investigated in humans.

A significant positive correlation between the levels of periostin and NAFLD US grades was observed in the orlistat group. Hence, the effect of orlistat on grades of fatty liver might be due to indirect downregulation of periostin, which needs to be confirmed and elaborated in further studies.

A remarkable upregulation of adiponectin levels was observed in NAFLD patients, which is consistent with the conclusion of a recent systemic review. ${ }^{20}$ In line with the previous studies, ${ }^{34,39}$ this study found a positive correlation of increased adiponectin level with weight loss in orlistat group supporting the assumption that adiponectin may be under a feedback regulation by reduction in body weight. ${ }^{40}$ It was also noted that there was a positive correlation between increased adiponectin levels and the degree of reduction in NAFLD US grades in orlistat group, similar to the findings of Harrison et al. ${ }^{33}$ This study also supports the agreement of the previous study that upregulation of adiponectin levels might be a therapeutic approach for the management of NAFLD. ${ }^{19}$

In this study, orlistat was effective in decreasing serum TNF- $\alpha$ level but failed to show a significant effect on serum IL-6 levels when compared to the hypocaloric diet. Furthermore, the decrease in serum levels of TNF- $\alpha$ and IL- 6 was not significantly associated with the degree of reduction in US grading, which might be due to the early stage of fatty liver in NAFLD patients.

In the present study, except minimal adverse effects like gastrointestinal distress $(16 \%)$ and fecal incontinence (4\%), no other serious adverse effects were reported with orlistat treatment.

\section{Limitations}

The first limitation of the present study is small sample size, especially in various grades of fatty liver. Secondly, this study used US imaging for assessing grades of fatty liver in place of liver biopsy, the gold standard for the assessment of fatty liver.

\section{Conclusion}

Orlistat could be an effective treatment for NAFLD patients without fibrosis. This study reported a downregulating effect of orlistat on serum periostin level first time. Furthermore, the reversal of fatty infiltration is positively associated with the downregulation of serum periostin and the upregulation of adiponectin after 16 weeks of orlistat treatment. Further clinical studies with different treatment modalities are needed to explore that downregulating periostin level might be an effective treatment strategy for improvement in fatty liver.

\section{Acknowledgments}

We express special thanks to Dr RM Pandey Professor and Head, AIIMS, New Delhi, for help in statistical analysis especially in the calculation of sample size for this study. We also extend our greatest thanks to the nursing and pathology staff of the Department of Medicine and Department of Radiology, Hamdard Institute of Medical Sciences \& Research, Jamia Hamdard, New Delhi, India, for providing their expert clinical assistance. Sun Pharma, India, provided partial assistance in the form of stipend, under the joint collaboration for the $\mathrm{PhD}$ program with Jamia Hamdard, New Delhi, India.

\section{Author contributions}

All authors contributed toward data analysis, drafting and revising the paper and agree to be accountable for all aspects of the work.

\section{Disclosure}

The authors report no conflicts of interest in this work.

\section{References}

1. Calzadilla Bertot L, Adams LA. The natural course of non-alcoholic fatty liver disease. Int J Mol Sci. 2016;17(5):774.

2. Younossi ZM, Koenig AB, Abdelatif D, Fazel Y, Henry L, Wymer M. Global epidemiology of non-alcoholic fatty liver disease - meta-analytic assessment of prevalence, incidence and outcomes. Hepatology. 2016; 64(1):73-84.

3. Kalra S, Vithalani M, Gulati G, et al. Study of prevalence of nonalcoholic fatty liver disease (NAFLD) in type 2 diabetes patients in India (SPRINT). J Assoc Physicians India. 2013;61(7):448-453.

4. Tolman KG, Dalpiaz AS. Treatment of non-alcoholic fatty liver disease. Ther Clin Risk Manag. 2007;3(6):1153-1163.

5. Valenti L, Bugianesi E, Pajvani U, Targher G. Nonalcoholic fatty liver disease: cause or consequence of type 2 diabetes? Liver Int. 2016;36(11): $1563-1579$.

6. Dongiovanni P, Anstee QM, Valenti L. Genetic predisposition in NAFLD and NASH: impact on severity of liver disease and response to treatment. Curr Pharm Des. 2013;19(29):5219-5238. 
7. Li L, Liu DW, Yan HY, Wang ZY, Zhao SH, Wang B. Obesity is an independent risk factor for non-alcoholic fatty liver disease: evidence from a meta-analysis of 21 cohort studies. Obes Rev. 2016;17(6):510-519.

8. Makris A, Foster GD. Dietary approaches to the treatment of obesity. Psychiatr Clin North Am. 2011;34(4):813-827.

9. Dajani A, AbuHammour A. Treatment of nonalcoholic fatty liver disease: where do we stand? an overview. Saudi J Gastroenterol. 2016; 22(2):91-105.

10. Filippatos TD, Derdemezis CS, Gazi IF, Nakou ES, Mikhailidis DP, Elisaf MS. Orlistat-associated adverse effects and drug interactions: a critical review. Drug Saf. 2008;31(1):53-65.

11. Athyros VG, Giouleme O, Ganotakis ES, et al. Safety and impact on cardiovascular events of long-term multifactorial treatment in patients with metabolic syndrome and abnormal liver function tests: a post hoc analysis of the randomised ATTEMPT study. Arch Med Sci. 2011;7(5): 796-805.

12. Harrison S, Fincke C, Helinski D, Torgerson S, Hayashi P. A pilot study of orlistat treatment in obese, non-alcoholic steatohepatitis patients. Aliment Pharmacol Ther. 2004;20(6):623-628.

13. Hussein O, Grosovski M, Schlesinger S, Szvalb S, Assy N. Orlistat reverse fatty infiltration and improves hepatic fibrosis in obese patients with nonalcoholic steatohepatitis (NASH). Dig Dis Sci. 2007; 52(10):2512-2519.

14. Zelber-Sagi S, Kessler A, Brazowsky E, et al. A double-blind randomized placebo-controlled trial of orlistat for the treatment of nonalcoholic fatty liver disease. Clin Gastroenterol Hepatol. 2006;4(5):639-644.

15. Lu Y, Liu X, Jiao Y, et al. Periostin promotes liver steatosis and hypertriglyceridemia through downregulation of PPAR $\alpha$. J Clin Invest. 2014;124(8):3501-3513.

16. Huang Y, Liu W, Xiao H, et al. Matricellular protein periostin contributes to hepatic inflammation and fibrosis. Am J Pathol. 2015;185(3): 786-797.

17. Amara S, Lopez K, Banan B, et al. Synergistic effect of pro-inflammatory TNF $\alpha$ and IL-17 in periostin mediated collagen deposition: potential role in liver fibrosis. Mol Immunol. 2015;64(1):26-35.

18. Zhu JZ, Zhu HT, Dai YN, et al. Serum periostin is a potential biomarker for non-alcoholic fatty liver disease: a case-control study. Endocrine. 2016;51(1):91-100.

19. Finelli C, Tarantino G. What is the role of adiponectin in obesity related non-alcoholic fatty liver disease? World J Gastroenterol. 2013;19(6): 802-812.

20. Derosa G, Maffioli P, Sahebkar A. Improvement of plasma adiponectin, leptin and C-reactive protein concentrations by orlistat: a systematic review and meta-analysis. Br J Clin Pharmacol. 2016;81(5):819-834.

21. Valenti L, Rametta R, Ruscica M, et al. The I148M PNPLA3 polymorphism influences serum adiponectin in patients with fatty liver and healthy controls. BMC Gastroenterol. 2012;12(1):111.

22. Wieckowska A, Papouchado BG, Li Z, Lopez R, Zein NN, Feldstein AE Increased hepatic and circulating interleukin-6 levels in human nonalcoholic steatohepatitis. Am J Gastroentero. 2008;103(6):1372-1379.

23. Bastard JP, Maachi M, Lagathu C, et al. Recent advances in the relationship between obesity, inflammation, and insulin resistance. Eur Cytokine Netw. 2006;17(1):4-12.

24. Wullaert A, van Loo G, Heyninck K, Beyaert R. Hepatic tumor necrosis factor signaling and nuclear factor- $\kappa \mathrm{B}$ : effects on liver homeostasis and beyond. Endocr Rev. 2007;28(4):365-386.
25. Flechtner-Mors M, George SN, Oeztuerk S, et al. Association of adiponectin with hepatic steatosis: a study of 1,349 subjects in a random population sample. BMC Res Notes. 2014;7:207.

26. World Medical Association. World Medical Association Declaration of Helsinki: ethical principles for medical research involving human subjects. JAMA. 2013;310(20):2191-2194.

27. von Elm E, Altman DG, Egger M, Pocock SJ, Gotzsche PC, Vandenbroucke JP. The strengthening the reporting of observational studies in epidemiology [STROBE] statement: guidelines for reporting observational studies. Gaceta sanitaria/S.E.S.P.A. 2008;22:144-150. Spanish.

28. Matthews D, Hosker J, Rudenski A, Naylor BA, Treacher DF, Turner RC. Homeostasis model assessment: insulin resistance and $\beta$-cell function from fasting plasma glucose and insulin concentrations in man. Diabetologia. 1985;28(7):412-419.

29. Roza AM, Shizgal HM. The Harris Benedict equation reevaluated: resting energy requirements and the body cell mass. Am J Clin Nutr. 1984; 40(1):168-182.

30. Scatarige JC, Scott WW, Donovan PJ, Siegelman SS, Sanders RC. Fatty infiltration of the liver: ultrasonographic and computed tomographic correlation. J Ultrasound Med. 1984;3(1):9-14.

31. Angulo P, Hui JM, Marchesini G, et al. The NAFLD fibrosis score: a noninvasive system that identifies liver fibrosis in patients with NAFLD. Hepatology. 2007;45(4):846-854.

32. Harrison SA, Oliver D, Arnold HL Gogia S, Neuschwander-Tetri BA. Development and validation of a simple NALFD clinical scoring system for identifying patient without advanced disease. Gut. 2008;57(10): 1441-1447.

33. Harrison SA, Fecht W, Brunt EM, Neuschwander-Tetri BA. Orlistat for overweight subjects with nonalcoholic steatohepatitis: a randomized, prospective trial. Hepatology. 2009;49(1):80-86.

34. Esposito K, Pontillo A, Di Palo C, et al. Effect of weight loss and lifestyle changes on vascular inflammatory markers in obese women: a randomized trial. JAMA. 2003;289(14):1799-1804.

35. Pan JJ, Fallon MB. Gender and racial differences in nonalcoholic fatty liver. World J Hepatol. 2014;6(5):274-283.

36. Luo Y, Qu H, Wang H, et al. Plasma periostin levels are increased in Chinese subjects with obesity and type 2 diabetes and are positively correlated with glucose and lipid parameters. Mediators Inflamm. 2016; 2016:6423637.

37. Dixon AN, Valsamakis G, Hanif MW, et al. Effect of the orlistat on serum endotoxin lipopolysaccharide and adipocytokines in South Asian individuals with impaired glucose tolerance. Int J Clin Pract. 2008; 62(7):1124-1129.

38. Lew WY, Bayna E, Molle ED, et al. Recurrent exposure to subclinical lipopolysaccharide increases mortality and induces cardiac fibrosis in mice. PLoS One. 2013;8(4):e61057.

39. Hsiao TJ, Wu LS, Huang SY, Lin E. A common variant in the adiponectin gene on weight loss and body composition under sibutramine therapy in obesity. Clin Pharmacol. 2010;2:105-110.

40. Yang WS, Lee WJ, Funahashi T, et al. Weight reduction increases plasma levels of an adipose-derived anti-inflammatory protein, adiponectin. J Clin Endocrinol Metab. 2001;86(8):3815-3819. 


\section{Supplementary material}

Table SI STROBE statement - checklist of items that should be included in reports of cohort studies

\begin{tabular}{|c|c|c|c|}
\hline Items & Item no & Recommendation & Page no(s) \\
\hline \multirow[t]{2}{*}{ Title and abstract } & 1 & (a) Indicate the study's design with a commonly used term in the title or the abstract & 1 \\
\hline & & $\begin{array}{l}\text { (b) Provide in the abstract an informative and balanced summary of what was done and what } \\
\text { was found }\end{array}$ & I \\
\hline \multicolumn{4}{|c|}{ (1) } \\
\hline Background/rationale & 2 & Explain the scientific background and rationale for the investigation being reported & 2 \\
\hline Objectives & 3 & State specific objectives, including any prespecified hypotheses & 2 \\
\hline \multicolumn{4}{|c|}{ 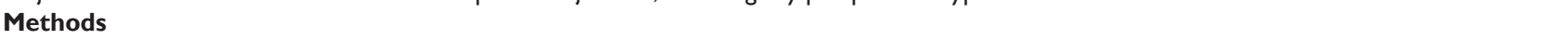 } \\
\hline Study design & 4 & Present key elements of study design early in the paper & 2 \\
\hline Setting & 5 & $\begin{array}{l}\text { Describe the setting, locations, and relevant dates, including periods of recruitment, exposure, } \\
\text { follow-up, and data collection }\end{array}$ & $2-4$ \\
\hline \multirow[t]{2}{*}{ Participants } & 6 & $\begin{array}{l}\text { (a) Give the eligibility criteria, and the sources and methods of selection of participants. } \\
\text { Describe methods of follow-up }\end{array}$ & $2-3$ \\
\hline & & (b) For matched studies, give matching criteria and number of exposed and unexposed & $\mathrm{N} / \mathrm{A}$ \\
\hline Variables & 7 & $\begin{array}{l}\text { Clearly define all outcomes, exposures, predictors, potential confounders, and effect modifiers. } \\
\text { Give diagnostic criteria, if applicable }\end{array}$ & $2-4$ \\
\hline $\begin{array}{l}\text { Data sources/ } \\
\text { measurement }\end{array}$ & 8 & $\begin{array}{l}\text { For each variable of interest, give sources of data and details of methods of assessment } \\
\text { (measurement). Describe comparability of assessment methods if there is more than one group }\end{array}$ & $2-4$ \\
\hline Bias & 9 & Describe any efforts to address potential sources of bias & $3-4$ \\
\hline Study size & 10 & Explain how the study size was arrived at & 2 \\
\hline Quantitative variables & 11 & $\begin{array}{l}\text { Explain how quantitative variables were handled in the analyses. If applicable, describe which } \\
\text { groupings were chosen and why }\end{array}$ & 4 \\
\hline Statistical methods & 12 & $\begin{array}{l}\text { (a) Describe all statistical methods, including those used to control for confounding } \\
\text { (b) Describe any methods used to examine subgroups and interactions } \\
\text { (c) Explain how missing data were addressed } \\
\text { (d) If applicable, explain how loss to follow-up was addressed } \\
\text { (e) Describe any sensitivity analyses }\end{array}$ & $\begin{array}{l}4 \\
4 \\
4 \\
\text { N/A } \\
4\end{array}$ \\
\hline \multicolumn{4}{|c|}{ (2) } \\
\hline \multirow[t]{3}{*}{ Participants } & $13 *$ & $\begin{array}{l}\text { (a) Report numbers of individuals at each stage of study - eg, numbers potentially eligible, } \\
\text { examined for eligibility, confirmed eligible, included in the study, completing follow-up, and } \\
\text { analysed }\end{array}$ & $3-4$ \\
\hline & & (b) Give reasons for nonparticipation at each stage & 4 \\
\hline & & (c) Consider use of a flow diagram & 4 \\
\hline \multirow[t]{3}{*}{ Descriptive data } & $14^{*}$ & $\begin{array}{l}\text { (a) Give characteristics of study participants (eg, demographic, clinical, social) and information } \\
\text { on exposures and potential confounders }\end{array}$ & 4 \\
\hline & & (b) Indicate number of participants with missing data for each variable of interest & 4 \\
\hline & & (c) Summarize follow-up time (eg, average and total amount) & 4 \\
\hline Outcome data & $15^{*}$ & Report numbers of outcome events or summary measures over time & $5-6$ \\
\hline \multirow[t]{3}{*}{ Main results } & 16 & $\begin{array}{l}\text { (a) Give unadjusted estimates and, if applicable, confounder-adjusted estimates and their } \\
\text { precision (eg, } 95 \% \text { confidence interval). Make clear which confounders were adjusted for and } \\
\text { why they were included }\end{array}$ & $5-6$ \\
\hline & & (b) Report category boundaries when continuous variables were categorized & $\mathrm{N} / \mathrm{A}$ \\
\hline & & $\begin{array}{l}\text { (c) If relevant, consider translating estimates of relative risk into absolute risk for a meaningful } \\
\text { time period }\end{array}$ & $\mathrm{N} / \mathrm{A}$ \\
\hline Other analyses & 17 & $\begin{array}{l}\text { Report other analyses done - eg, analyses of subgroups and interactions, and sensitivity } \\
\text { analyses }\end{array}$ & $5-6$ \\
\hline \multicolumn{4}{|c|}{$\ln _{1}$} \\
\hline Key results & 18 & Summarize key results with reference to study objectives & $6-8$ \\
\hline Limitations & 19 & $\begin{array}{l}\text { Discuss limitations of the study, taking into account sources of potential bias or imprecision. } \\
\text { Discuss both direction and magnitude of any potential bias }\end{array}$ & 8 \\
\hline Interpretation & 20 & $\begin{array}{l}\text { Give a cautious overall interpretation of results considering objectives, limitations, multiplicity } \\
\text { of analyses, results from similar studies, and other relevant evidence }\end{array}$ & $6-8$ \\
\hline $\begin{array}{l}\text { Generalizability } \\
\text { Other information }\end{array}$ & 21 & Discuss the generalizability (external validity) of the study results & 8 \\
\hline Funding & 22 & $\begin{array}{l}\text { Give the source of funding and the role of the funders for the present study and, if applicable, } \\
\text { for the original study on which the present article is based }\end{array}$ & 8 \\
\hline
\end{tabular}

Notes: *Give information separately for exposed and unexposed groups. An Explanation and Elaboration article discusses each checklist item and gives methodological background and published examples of transparent reporting. The STROBE checklist is best used in conjunction with this article. Information on the STROBE Initiative is

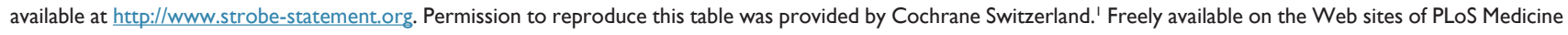
at http://www.plosmedicine.org/, Annals of Internal Medicine at http://www.annals.org/, and Epidemiology at http://www.epidem.com/.

Abbreviations: N/A, not applicable; STROBE, Strengthening the Reporting of Observational Studies in Epidemiology. 


\section{Reference}

1. STROBE statement. Available from: http://www.strobe-statement.org/ index.php?id=available-checklists. Accessed February 1, 2017.

\section{Publish your work in this journal}

Therapeutics and Clinical Risk Management is an international, peerreviewed journal of clinical therapeutics and risk management, focusing on concise rapid reporting of clinical studies in all therapeutic areas, outcomes, safety, and programs for the effective, safe, and sustained use of medicines. This journal is indexed on PubMed Central, CAS,
EMBase, Scopus and the Elsevier Bibliographic databases. The manuscript management system is completely online and includes a very quick and fair peer-review system, which is all easy to use. Visit http://www.dovepress.com/testimonials.php to read real quotes from published authors.

Submit your manuscript here: http://www.dovepress.com/therapeutics-and-clinical-risk-management-journal 